\title{
The impulse response of a high-speed jet forced with localized arc filament plasma actuators
}

Aniruddha Sinha, Hind Alkandry, Martin Kearney-Fischer, Mo Samimy, and Tim Colonius

Citation: Phys. Fluids 24, 125104 (2012); doi: 10.1063/1.4772191

View online: http://dx.doi.org/10.1063/1.4772191

View Table of Contents: http://pof.aip.org/resource/1/PHFLE6/v24/i12

Published by the American Institute of Physics.

\section{Related Articles}

Experimental study of planar opposed jets with acoustic excitation Phys. Fluids 25, 014108 (2013)

Similarity analysis of the momentum field of a subsonic, plane air jet with varying jet-exit and local Reynolds numbers

Phys. Fluids 25, 015115 (2013)

Electrohydrodynamic printing under applied pole-type nozzle configuration

Appl. Phys. Lett. 102, 024101 (2013)

Effects of fluid properties and laser fluence on jet formation during laser direct writing of glycerol solution J. Appl. Phys. 112, 083105 (2012)

Bag instabilities

Phys. Fluids 24, 091112 (2012)

\section{Additional information on Phys. Fluids}

Journal Homepage: http://pof.aip.org/

Journal Information: http://pof.aip.org/about/about_the_journal

Top downloads: http://pof.aip.org/features/most_downloaded

Information for Authors: http://pof.aip.org/authors

\section{ADVERTISEMENT}

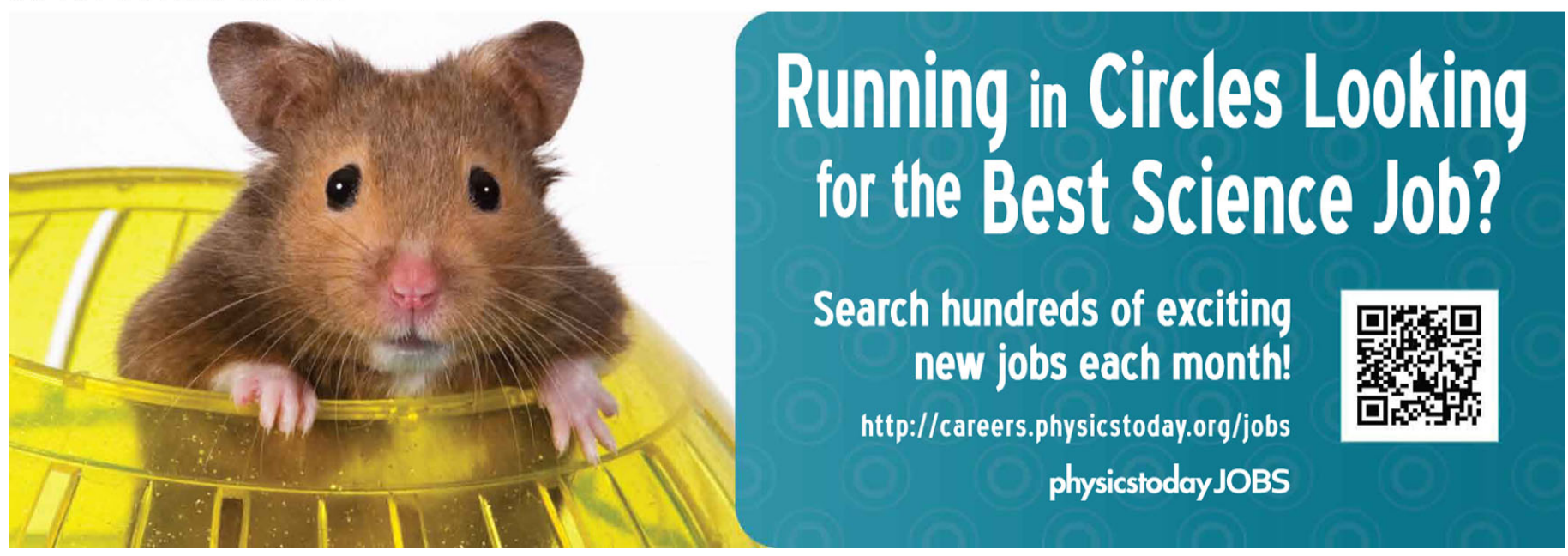




\title{
The impulse response of a high-speed jet forced with localized arc filament plasma actuators
}

\author{
Aniruddha Sinha, ${ }^{1}$ Hind Alkandry, ${ }^{2}$ Martin Kearney-Fischer, ${ }^{3}$ \\ Mo Samimy, ${ }^{2, \text { a) }}$ and Tim Colonius ${ }^{1}$ \\ ${ }^{1}$ California Institute of Technology, Pasadena, California 91106, USA \\ ${ }^{2}$ Ohio State University, Columbus, Ohio 43235, USA \\ ${ }^{3}$ Lockheed Martin Aeronautics, Palmdale, California 93599, USA
}

(Received 16 August 2012; accepted 8 November 2012; published online 19 December 2012)

\begin{abstract}
We present experimental and theoretical analyses of the response of high-speed, highReynolds-number, round jets to impulsive forcing with arc-filament-plasma actuators. The impulse response is obtained with forcing Strouhal numbers, based on the nozzle exit diameter and exit center line velocity, less than 0.1 . The resulting phase-averaged near-field pressure signature displays a compact wave with a positive peak preceding a negative one, indicative of a large scale structure in the shear layer of the jet. Scaling laws derived by operating the jet at four subsonic Mach numbers are used to distinguish this hydrodynamic component of the phase-averaged jet response from the direct actuator noise. As the forcing frequency increases, the compact waves in the near-field pressure signal overlap each other, indicating interaction of the growing seeded structures. For this regime, the phase-averaged response is approximately replicated by linear superposition of the impulse response, thereby demonstrating the quasi-linearity of structure interaction. A novel application of linear parabolized stability theory yields a successful model of the impulse response. (c) 2012 American Institute of Physics. [http://dx.doi.org/10.1063/1.4772191]
\end{abstract}

\section{INTRODUCTION}

Active flow control has been previously applied to high-speed and high Reynolds number jets to achieve noise mitigation and mixing enhancement. In addition to these practical goals, the organization of the flow afforded by periodic forcing has been used as a diagnostic aid to facilitate understanding of turbulence, and we contribute to this effort here.

Localized arc filament plasma actuators (LAFPAs) have been developed and implemented for active control of jets at the Gas Dynamics and Turbulence Laboratory in Ohio State University over almost a decade. ${ }^{1-3}$ Each LAFPA consists of a pair of electrodes placed very near the nozzle exit, connected to a high voltage source through a switching circuitry. Closing the switch causes the voltage across the electrodes to rise until the air between them undergoes breakdown, thereby injecting a perturbation to the flow. Controlling the switching periodicity and the firing pattern of the LAFPAs arranged around the nozzle exit periphery allows metered injection of high-amplitude perturbations of various frequencies and azimuthal modes into the flow.

The shear layer of the jet is receptive to small unsteady perturbations at the nozzle exit. Such perturbations can grow into large scale turbulence structures (LSS, henceforth) via natural instabilities of the shear layer. ${ }^{4,5}$ This allows plasma actuation to manipulate and organize LSS in the jet shear layer with periodicity (in both time and azimuth) closely matching the prescribed excitation ${ }^{3,6-9}$ at relatively low energy expense. ${ }^{2}$ The LAFPAs have thereby been proven effective for both mixing enhancement and noise reduction over a large range of Mach numbers and temperatures (see Ref. 10 for a recent review).

a) Author to whom correspondence should be addressed. Electronic mail: samimy.1@osu.edu. 
The performance of LAFPAs has been characterized with several measurement techniques, viz., particle image velocimetry (PIV), schlieren imaging, flow visualization, and far-field acoustic measurements. The pressure in the irrotational near field (NFP, henceforth) has also been assayed, ${ }^{11}$ but not as exhaustively as the other investigations listed. The present research uses phase-averaged NFP measurements to further characterize the effect of LAFPA forcing on jets. By positioning the probes just outside the shear layer, we obtain a non-intrusive measurement of the hydrodynamic pressure field that is linearly driven by large-scale structures. ${ }^{12-17}$ This avoids the strong nonlinear vorticity and entropy disturbances inside the shear layer, as well as the acoustic waves that dominate further outward. ${ }^{12,15}$ Plasma actuation manipulates the LSS, so that the forcing effect can be conveniently assessed by analyzing the NFP.

Previous attempts at unsteady excitation of jets have used acoustic drivers (e.g., Ref. 4), piezoelectric synthetic jets, ${ }^{18}$ and fluidic injection (e.g., Refs. 19 and 20). The perturbations injected into the flow by these actuators are typically characterized by smooth waveforms with independent control of the amplitude. The distinguishing feature of the LAFPAs is the impulsive nature of the breakdown that injects the perturbation at an amplitude that is fixed by the operating condition. This allows a study of the impulse response of the jet. Apart from offering a window into the details of the control mechanisms at play, the knowledge of the impulse response is also crucial for model design aimed at feedback control. It is shown here that the impulse response is essentially the response of the jet to very low-frequency operation of the LAFPAs, since the seeded perturbations evolve independently in this forcing regime. With higher frequencies of LAFPA pulsation, the imposed periodicity affects the interaction and development of individual perturbations.

We hypothesize that each impulse of the LAFPA seeds a perturbation in the initial shear layer that grows and rolls up into a LSS. This premise is investigated by deriving scaling laws from the phase-averaged responses of jets over a range of subsonic Mach numbers forced at a large range of frequencies. The high-amplitude impulsive flow perturbations generated by LAFPAs may have been expected to cause a strongly nonlinear effect on the jet. However, the theory of linear parabolized stability equations (PSE) $)^{21}$ is shown to be a reasonably accurate model of the impulse response of the jet herein. This follows the recent success of PSE in predicting the characteristics of harmonic wavepackets in the flow field and hydrodynamic near field of unforced subsonic and supersonic turbulent jets. ${ }^{22-24}$ We mention that the impulse response of a Mach 2.5 jet has been computed with global mode analysis previously, ${ }^{25}$ but experimental validation has been lacking in this case.

The article is organized as follows. Section II documents the experimental setup. Section III briefly introduces the PSE theory. Section IV characterizes the NFP of the unforced jet to establish a baseline, and also discusses the modeling of some aspects of the NFP using PSE. Section V details the impulse response of the jet, and demonstrates the applicability of linear PSE for predicting it. The effect of the periodicity of impulsive actuation is explored in depth in Sec. VI to reveal a quasi-linear property. Section VII concludes with a discussion of the implications of this research.

\section{EXPERIMENTAL SETUP}

\section{A. Test facility}

All the experiments are conducted in the newly upgraded anechoic chamber at Ohio State University. A schematic of the chamber is shown in Figure 1; its design has been validated and documented in Ref. 26. Ambient air is compressed using three 5-stage reciprocating compressors, filtered, dried, and stored in two $36 \mathrm{~m}^{3}$ tanks at up to $16 \mathrm{MPa}$. The air is discharged horizontally through the nozzle into the anechoic chamber, and then through an exhaust system to the outdoors.

The present work employs a thick-lipped converging axisymmetric nozzle with exit diameter $D$ of $25.4 \mathrm{~mm}$. The internal contour of the nozzle is designed using a fifth-order polynomial. The nozzle is operated at jet Mach numbers $\left(M_{j}\right)$ of $0.80,0.85,0.90$, and 0.95 . The jets are not heated, and the stagnation temperature typically stays between $5{ }^{\circ} \mathrm{C}$ and $15^{\circ} \mathrm{C}$. The Reynolds number based on $D$ and the exit conditions ranges between $5.6 \times 10^{5}$ and $7.1 \times 10^{5}$. Previous hot-wire measurements indicate that the shear layer is turbulent at the nozzle exit with initial momentum thickness $\approx 0.09 \mathrm{~mm}$ and initial shear layer thickness (the thickness of the boundary layer exiting the nozzle) $\approx 1 \mathrm{~mm}^{7}$ 


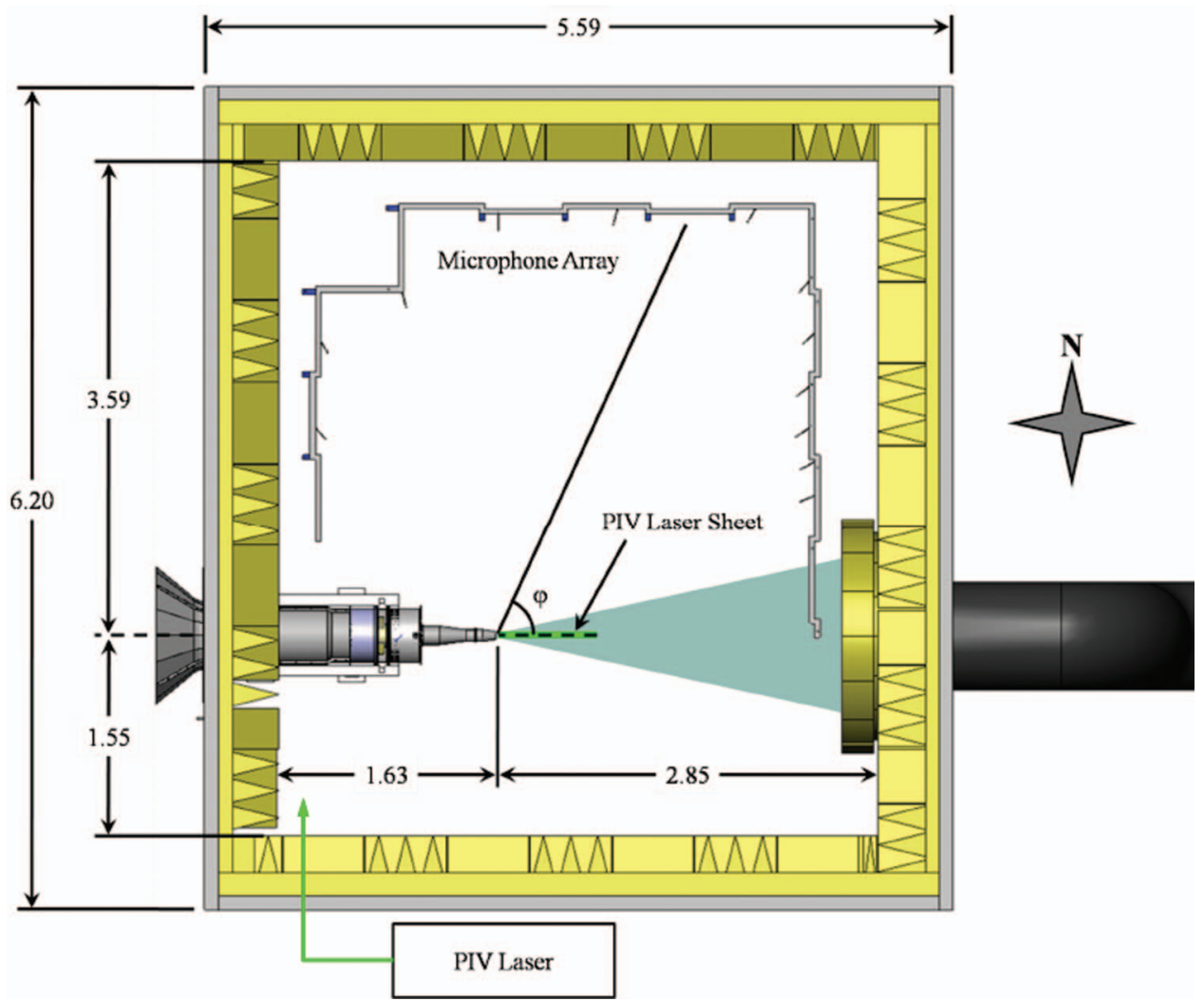

FIG. 1. Schematic of the anechoic chamber and jet with measurement tools. Dimensions are in metres.

Figure 2 depicts the mean axial velocity field of the unforced $M_{j}=0.9$ jet as measured in an earlier PIV assay. ${ }^{7}$ The effect of the thick lip, needed to install the plasma actuators, is observed in the thickness of the initial shear layer. The length of the potential core is $\approx 6 D$.

\section{B. Localized arc filament plasma actuators}

A LAFPA consists of a pair of pin electrodes held in place using a nozzle extension (see Figure 3). Eight such actuators are uniformly distributed around the nozzle perimeter, approximately $1 \mathrm{~mm}$ upstream of the nozzle extension exit plane. A ring groove of $0.5 \mathrm{~mm}$ depth and $1 \mathrm{~mm}$ width is used to house the electrodes and to shield the plasma. The groove does not have a significant effect on the baseline or the controlled jet. ${ }^{9}$ The nozzle extension is made of boron nitride and tungsten wires of $1 \mathrm{~mm}$ diameter are used for electrodes. The spacing between the pair of electrodes in an actuator is $4 \mathrm{~mm}$, measured center-to-center at the arcing tips.

A second-generation eight-channel high-voltage dc plasma generator, designed and built inhouse, is used to drive the actuators. The circuitry powering each LAFPA consists of a transformer with a switchable capacitor on the primary side and the arcing electrodes connected across the

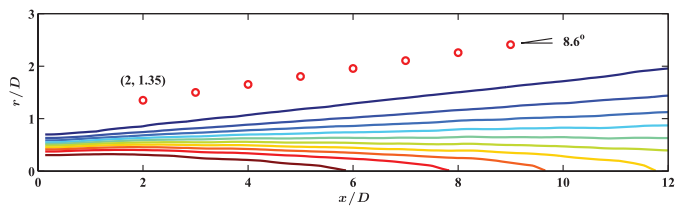

FIG. 2. Mean axial velocity field of unforced $M_{j}=0.9$ jet, normalized by $U_{j}$ (contour levels are $0.05-0.95$ in equal increments). Positions of pressure sensors are indicated. 


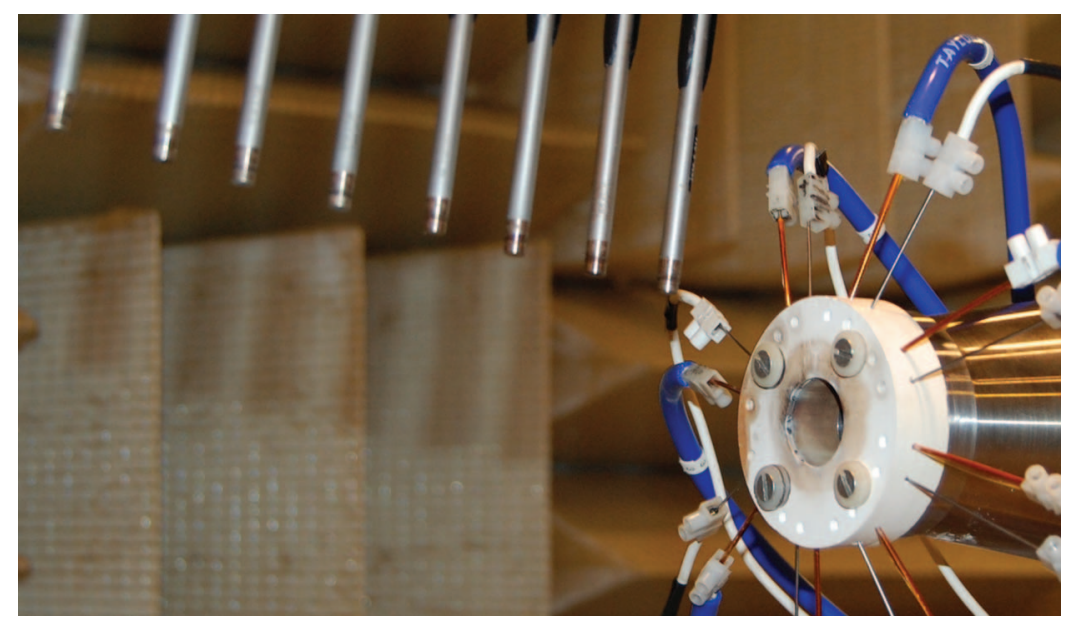

FIG. 3. LAFPAs housed in the ceramic nozzle extension, and the linear array of microphones for measuring the near-field pressure.

secondary coil. The capacitor is charged to $100 \mathrm{~V}$ with the switch open. A rectangular pulse from the controlling computer then closes the switch for $7 \mu \mathrm{s}$, thereby discharging the capacitor through the primary coil of the transformer. The secondary voltage increases until it is high enough to overcome the resistance of the electrode gap and a plasma generating arc is formed. This arc has a duration of $\approx 6 \mu$ s beyond the trigger turn off while the transformer de-energizes. This cycle can repeat up to $100 \mathrm{kHz}$, but the cooling circuitry (being upgraded) currently limits the operation to $20 \mathrm{kHz}$.

The actuator system allows independent control of firing frequency as well as the order of firing around the periphery (azimuthal mode). In the present experiments, all eight actuators are fired synchronously, thereby simulating axisymmetric forcing (i.e., $m=0$ azimuthal mode). The forcing frequency is varied between $250 \mathrm{~Hz}$ and $15 \mathrm{kHz}$. Based on previous studies of the effect of varying the pulse width, ${ }^{9}$ it is fixed at $7 \mu \mathrm{s}$ in these experiments, as mentioned above. This is not the time-scale of impulsive perturbation, which is determined by the nanosecond-scale electric breakdown. Note that the LAFPA system does not admit direct control of the amplitude of the perturbations injected.

\section{Data acquisition}

\section{Near-field pressure measurements}

The NFP is acquired using a linear array of eight microphones in a meridional plane of the jet, as depicted in Figure 3. The configuration is fully specified in Figure 2. The linear configuration of the array and the axisymmetry of the domain allow the sole specification of the axial coordinate of a sensor to fully determine its spatial location. The 1/4 in. Bruel and Kjaer (B\&K) (model 4939) microphones are paired with $B \& K$ pre-amplifiers (model 2670). The excitation signal to the microphones, as well as the amplification and filtering of the output, are performed on B\&K Nexus signal conditioners (model 2690). The circuitry of the microphones was found to be robust to the electro-magnetic interference arising from the LAFPAs.

The voltage signal from each microphone is bandpass filtered between $20 \mathrm{~Hz}$ and $100 \mathrm{kHz}$. The amplified signals are simultaneously acquired using National Instruments (NI) PXI-6133 A/D boards and LabVIEW software. The microphones are calibrated using a $114 \mathrm{~dB}, 1 \mathrm{kHz}$ sine wave, and the frequency response of the microphones is flat up to $80 \mathrm{kHz}$ with the microphone grid cover removed. Signals are acquired at $200 \mathrm{kHz}$ with 81920 data points per block of samples. Ten such blocks of data are recorded for each experimental case resulting in $4 \mathrm{~s}$ worth of data, which is sufficient for convergence of turbulence statistics. Unless mentioned otherwise, the short-time window is 
set to 8192 samples for calculation of power spectral densities, resulting in a spectral resolution of $24.4 \mathrm{~Hz}$.

\section{Actuation phase}

The phase-averaging of the forcing response requires precise knowledge of the actuation signal corresponding to each pressure sample. In each experimental run with forcing, a unique forcing frequency is employed and all the LAFPAs are fired simultaneously. The particular character of LAFPAs described above then means that the only unknown forcing information at any time is its phase. A sampling frequency of the order of $\mathrm{MHz}$ would have been required to determine the phase from the rectangular pulse train controlling the LAFPA operation. Instead, the pulse train controlling the first LAFPA is supplied to an Agilent 3320A $20 \mathrm{MHz}$ arbitrary waveform generator, where each of the square rising edges on the rectangular pulse train triggers a rising ramp signal (an example appears subsequently in Figure 7). This sequence of ramp signals is acquired simultaneously with the pressure signals, and decoded in post-processing to determine the actuation phase at each pressure sample.

\section{MODELING JET ACTUATION USING PARABOLIZED STABILITY EQUATIONS}

Flow stability theory attempts to predict the evolution of perturbations, whether natural or artificially seeded. Parabolized stability equations, a flavor of spatial stability theory, is invoked here to model the response of the highly turbulent jet to perturbations seeded by the LAFPAs. Weakly non-parallel theory improves on classical parallel flow analysis, and PSE is a fast approximation of the former that is valid for convectively unstable flows, like jets. PSE was originally developed to predict boundary layer transition. ${ }^{21}$ Subsequently, it was adapted for analyzing the stability of laminar and transitional shear flows (e.g., Ref. 27). In the above approaches, the perturbations were modeled as flow fluctuations about a laminar base flow. Recently, the theory has been successfully applied to highly turbulent jets by choosing the time-averaged mean flow as the base flow. ${ }^{22-24}$ These last cited articles can be referred for the details of the theory which is briefly sketched here.

The governing conservation equations are written in terms of pressure, specific volume, and the three velocity components in cylindrical coordinates. ${ }^{23}$ These non-dimensionalized flow quantities, generally denoted by $Q$, are decomposed into fluctuations $q$ about the time-averaged or ensembleaveraged mean flow $\bar{Q}$. Since the flow is statistically stationary and axisymmetric, $q$ is approximately expanded into a finite number of discrete frequencies and azimuthal Fourier modes. The Fourier modes are further decomposed into a slowly varying shape function and a rapidly varying wave-like part in the axial direction to arrive at the ansatz

$$
q(x, r, \theta, t)=\sum_{n=-N}^{N} \sum_{m=-M}^{M} \hat{q}_{m n}(x, r) \exp \left\{\mathrm{i}\left(\int_{x_{0}}^{x} \alpha_{m n}(\xi) \mathrm{d} \xi+m \theta-n \omega_{0} t\right)\right\} .
$$

Here, $\hat{q}_{m n}$ is the modal shape function, $\omega_{0}$ is the smallest circular frequency retained in the expansion, and $m$ and $n$, respectively, denote the azimuthal mode number and frequency harmonic. The real and imaginary parts of $\alpha_{m n}$ are, respectively, the axial wavenumber $\alpha_{m n}^{r}$ and growth rate $\alpha_{m n}^{i}$. The initial axial station, typically taken to be near the nozzle exit, is denoted by $x_{0}$.

Substitution of the above ansatz in the governing equations, and neglect of second derivatives of fluctuations in the axial direction under the assumption of slow axial variations, leads to the following system of equations:

$$
\left[\mathcal{L}_{0}+\mathcal{L}_{x} \frac{\partial}{\partial x}+\mathcal{L}_{r} \frac{\partial}{\partial r}+\mathcal{L}_{r r} \frac{\partial^{2}}{\partial r^{2}}+\mathcal{L}_{x r} \frac{\partial^{2}}{\partial x \partial r}\right] \hat{q}_{m n}=\mathcal{F}_{m n}
$$

The linear operators $\mathcal{L}$ on the left-hand side are functions of $\bar{Q}, m, n, \alpha_{m n}$, and $\omega_{0}$, in addition to the Reynolds number, Mach number, and Prandtl number of the flow. ${ }^{23}$ The nonlinearities are formally collected in the right-hand side term $\mathcal{F}_{m n}$. Following the success of linear PSE in modeling the fluctuations in the flow field and hydrodynamic near field of subsonic and supersonic turbulent jets, ${ }^{22-24}$ 
the nonlinearities are neglected in the present model too. The above equations are complemented by an additional integral constraint that disambiguates the apportionment of the axial variation of the modes between the shape function and the wave-like part. ${ }^{22}$ The boundary conditions in the radial direction are described in Ref. 23.

The parabolization is known to be imperfect (e.g., Ref. 28), but a suitably coarse axial step size $\Delta x$ leads to a stable marching scheme. Thus, one must specify the $\hat{q}_{m n}$ and $\alpha_{m n}$ at the first axial station $x=x_{0}$ to initiate the solution. An adequate procedure is to use the Kelvin-Helmholtz (K-H) mode (the only unstable mode in subsonic jets) from the quasi-parallel linear stability problem solved at this station. ${ }^{22,23}$

The PSE approach is first used in Sec. IV B to analyze the unforced jet, using the mean flow field obtained from PIV (as in Figure 2). The mean thermodynamic quantities are estimated from the mean axial velocity field. ${ }^{22}$ Subsequently, the PSE modes are used to model the impulse response of the jet forced with LAFPAs in Sec. V B. Further description of the model is deferred until the action of the LAFPAs is elucidated with the experimental results.

\section{UNFORCED JET RESULTS}

A preliminary characterization of the near-field pressure in the unforced jet situates the ensuing discussion of the forcing response. The availability of data over the range of nozzle exit velocities also affords a unique opportunity to evaluate some of the scaling laws commonly used in NFP analysis. Analysis of the experimental results is presented in Sec. IV A. The PSE model for the same is described in Sec. IV B.

\section{A. Experimental observations}

The established normalization for the near-field pressure fluctuation, $p$, at least for cold subsonic jets, is the nozzle exit dynamic head $\rho_{j} U_{j}^{2}$, where $\rho_{j}$ and $U_{j}$ are the exit centerline density and axial velocity, respectively. ${ }^{12,29}$ This scaling is implied for all NFP data presented. The NFP spectral frequency, $f$, is always converted to the non-dimensional Strouhal number, $S t_{D}=f D / U_{j}$. The amplitude and characteristic frequency of the NFP are functions of the measurement location, but these are not addressed in the above scaling. The axial distance, $x$, from the nozzle exit plane and the radial distance, $r$, from the jet centerline are normalized by $D$.

The mean-square (alternatively termed "energy") of the pressure fluctuations, $p_{\mathrm{MS}}$, in the unforced jets is presented in Figure 4. The nozzle exit dynamic head is nearly collapsing the data over this range of Mach numbers. The growth and decay of the NFP amplitude with axial distance has been well documented. ${ }^{13-15}$

A notion of convective velocity, $U_{c}$, of the LSS can be obtained from a space-time crosscorrelation of the NFP signals measured at two axial stations. ${ }^{13,14}$ The convective velocity is known to scale with the centerline velocity, and thus $U_{c}$ decreases downstream of the potential core. The correlation method is used to calculate $U_{c} / U_{j}$, and a value of 0.71 is obtained at $x=4 D$ for all four exit velocities tested. A value of 0.69 was also reported for a Mach 0.85 jet. $^{14}$

Figure 5(a) presents the power spectral density (PSD) plots for the unforced jets measured at $x$ $=4 D$. A good collapse of the data is observed with the scaling using Strouhal number and the nozzle

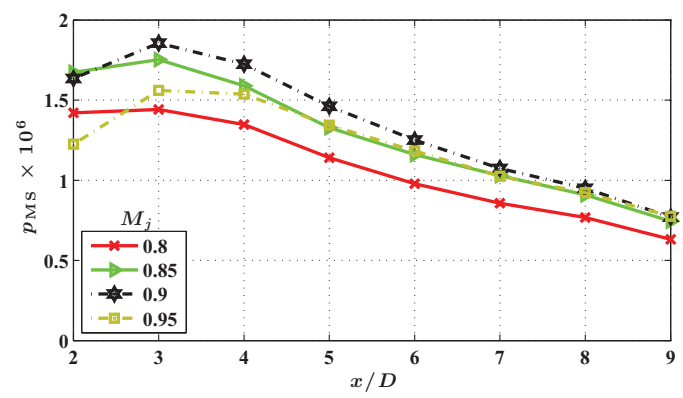

FIG. 4. Mean-square of near-field normalized pressure fluctuations in unforced jets. 


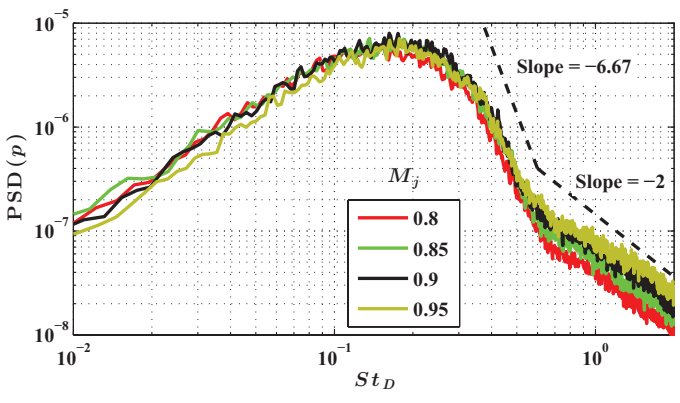

(a)

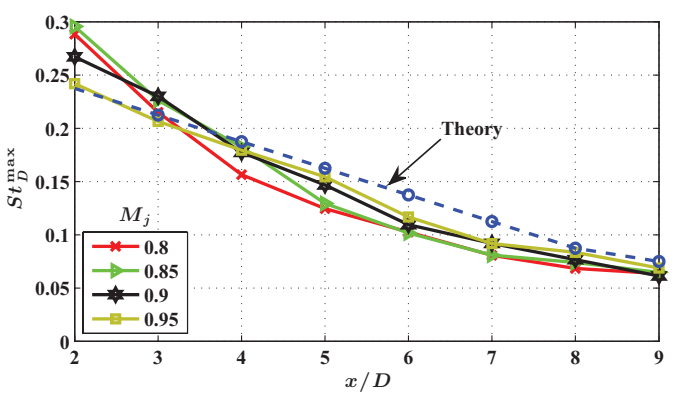

(b)

FIG. 5. Spectral character of NFP in unforced jets. (a) PSD of normalized pressure at $x=4 D$. (b) $S t_{D}$ at peak of PSD.

exit dynamic head. The change in the slope of the NFP spectrum, noted at $S t_{D} \approx 0.6$ in Figure 5(a), is indicative of the demarcation between the hydrodynamic and acoustic frequential components of the NFP. ${ }^{12}$ This critical frequency is typically reported in terms of $k y$. Here, $k=2 \pi f / a_{0}$ denotes the wavenumber, with $a_{0}$ being the ambient speed of sound. Also, $y$ refers to the radial distance of the NFP sensor from the lip-line, which is assumed to be an approximate location of the source of the pressure fluctuations. The critical $k y$ value reported in the literature varies considerably and is approximately 2 for low subsonic jets, ${ }^{12,29,30}$ and 3.5 for a Mach 0.85 jet. ${ }^{31,32}$ The $S t_{D} \approx 0.6$ demarcation in the spectra presented in Figure 5(a) translates to ky values between 3.3 and 3.8 for the range of Mach numbers studied here, which agrees well with results in the literature.

A better collapse of the critical frequency may be obtained with $f y / U_{c}$ than $k y$. Indeed, a consistent value of unity was observed at all measurement locations and Mach numbers considered here. This factor also turns out to be unity for the Mach 0.3 jet reported in the original article. ${ }^{12}$ By definition, $U_{c} / f$ is the axial wavelength of a structure of frequency $f$. Thus, the above scaling of the critical frequency indicates that the predominant character of the pressure associated with a structure is changing to acoustic at a radial distance approximately equal to its axial wavelength.

In Figure 5(a), the spectral change to acoustic character is occurring almost two orders-ofmagnitudes below the peak. Thus, the NFP is predominantly hydrodynamic at the location of the sensor array, and the spectral peak frequency reflects the temporal character of the most dominant large-scale structures. Figure 5(b) presents the Strouhal number at the spectral peak, $S t_{D}^{\max }$, over the range of measurement locations and jet operating conditions. The decrease in $S t_{D}^{\max }$ with axial distance from the exit has been discussed in the literature, ${ }^{12-14}$ and this has been linked to the growth of LSS resulting in shear layer growth. In particular, Ref. 12 noted that multiplying the spectral frequency by $\left(x-x_{0}\right)$ can collapse the spectra between $x \approx 3 D$ and the end of the potential core due to the self-similarity in this range. Here, $x_{0}$ is a fitting constant. A least-squares linear fit of $1 / S t_{D}^{\max }$ in the range $2 \leq x / D \leq 6$ yielded the following relation:

$$
\frac{1}{S t_{D}^{\max }}=1.40 x / D+0.53
$$

with coefficient of determination $R^{2}=0.92$. The theoretical curve in Figure 5(b) is obtained by PSE below.

\section{B. Comparison with PSE analysis}

The parabolized stability calculations are initiated with the Kelvin-Helmholtz mode obtained from the quasi-parallel linear stability analysis performed at $x=0.5 \mathrm{D}$, which is suitably close to the nozzle exit. The growth rates of the K-H instability for various pertinent Fourier modes in the $M_{j}$ $=0.9$ jet are presented in Figure 6(a). Azimuthal modes higher than 2 were found to be damped at all frequencies considered. The mean axial velocity field for the $M_{j}=0.9$ jet from the thick-lipped nozzle considered here (see Figure 2) has indicated the significantly thickened initial shear layer. This results in a substantial decrease in growth rates as well as a reduction in the range of unstable 


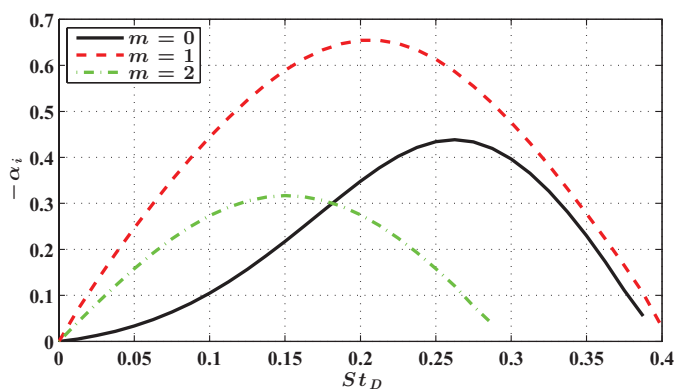

(a)

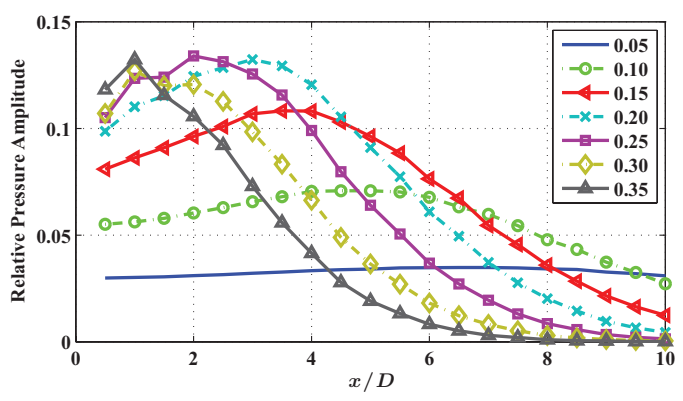

(b)

FIG. 6. PSE results for unforced $M_{j}=0.9$ jet. (a) Growth rates at $x=0.5 D$. (b) Relative pressure amplitudes for $m=0$.

frequencies, compared to thin-lipped jets. ${ }^{15}$ In particular, since all frequencies beyond $S t_{D}=0.4$ are damped at the initial axial station, they do not contribute to the convective instability and are neglected in the subsequent PSE computations.

Linear PSE systems, being homogenous, have arbitrarily scaled solutions. In order to compare relative amplitudes of different modes at downstream stations, it is necessary to specify a reasonable relation at the initial axial location. The azimuthal modal spectrum of the axial velocity fluctuations peaks between $m=3$ and $6^{33}$ near the nozzle lip for a turbulent jet. However, modes $m=0,1$, and 2 dominate the near field pressure, ${ }^{15}$ and are thus the only ones to be considered in a linear prediction of the same. Since the relative amplitudes of the different azimuthal modes of velocity were not measured for the jet under consideration here, no attempt will be made to compare their amplitudes in the near pressure field. On the other hand, the frequency spectrum of the axial velocity fluctuations near the nozzle lip for a turbulent jet is known to be flat up to $S t_{D} \approx 0.5 .{ }^{33,34}$ This observation is used to scale the PSE solutions for $m=0$ such that their axial velocity components are unity at $(x=0.5 D, r=0.5 D)$. The resulting pressure amplitudes for the different frequencies at the location of the near-field linear array are shown in Figure 6(b). This predicts that lower frequencies will dominate at downstream locations, a well-known fact that was also re-confirmed for the jets under consideration in Figure 5(b). The initial undulations in the pressure amplitudes reflect the uncertainty in specifying the correct mode shape at the initial station.

To be more quantitative, the theoretical curve displayed in Figure 5(b) is obtained by identifying the most amplified frequency at each axial station in Figure 6(b). This operation was performed using PSE solutions on a grid of frequencies that was four times finely resolved than that depicted. The theoretical $S t_{D}^{\max }$ curve for the $m=1$ PSE modes (not shown) was also very similar. Linear PSE has been demonstrated to be a very good model for the hydrodynamic pressure field in high subsonic jets. ${ }^{22}$ Figure 5(b) shows that the predictions from this theory match the experimental observations in the thick-lipped jets under consideration here too.

\section{THE IMPULSE RESPONSE}

The sudden electric breakdown that occurs in the air between the two electrodes with each pulse of the plasma actuator generates a delta function-like pressure response in its immediate vicinity. Although this could not be observed experimentally, it was suggested by a simple one-dimensional model of the arc filament. ${ }^{2}$ Further support for this phenomenon is provided by the sharpness of the compression waves observed in schlieren imagery. ${ }^{9,35}$ Assuming that the perturbation injected by the plasma actuator is indeed localized in space and time, we have an opportunity to study the impulse response of the jet.

Figure 7 depicts the relationship between the actuation and the near-field pressure at a representative location. It is clear that each LAFPA firing is triggering a pressure pulse that rises well above the background turbulence. The compactness of the response, which is analyzed in detail below, allows one to simulate the impulse with periodic forcing as long as the forcing frequency, $f_{F}$, 


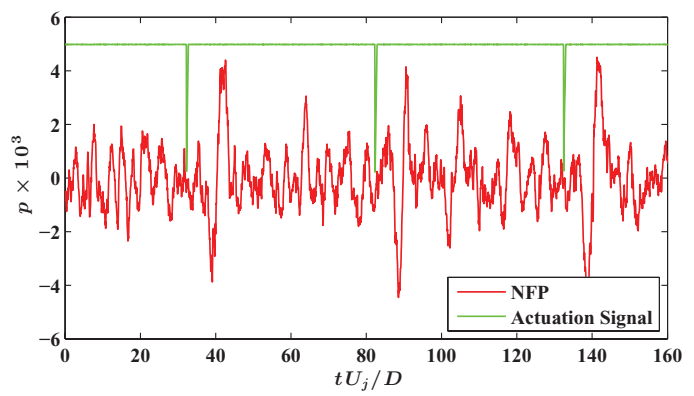

FIG. 7. Response of NFP at $x=4 D$ for the $M_{j}=0.9$ jet forced at $S t_{D F}=0.02$. The falling edges of the ramp signal indicate the instants at which the LAFPAs are switched on.

is sufficiently low. The consequent advantage is the opportunity for phase-averaging to isolate the impulse response from the chaos. The forcing Strouhal number $S t_{D F}\left(=f_{F} D / U_{j}\right)$ of 0.02 is shown to be appropriate for studying the impulse response, and this is used for all results presented in this section. The effect of increasing the forcing frequency is investigated in Sec. VI. This section is divided in two parts: Section V A describes the analysis of experimental observations of the response, and Sec. V B discusses a model of this behavior using linear PSE.

\section{A. Experimental observation of impulse response}

The triple decomposition ${ }^{36}$ is a convenient tool to analyze periodically forced flows. For the NFP, the mean value is the uniform ambient pressure, and thus the decomposition is simplified. The instantaneous fluctuating pressure is then written as

$$
p=\tilde{p}+p^{\prime}
$$

where $\tilde{p}$ is the "wave" component of the pressure, and $p^{\prime}$ is the residual fluctuations. For a given forcing frequency $f_{F}$, the wave component is computed by the following phase averaging technique:

$$
\tilde{p}\left(T ; f_{F}\right):=\lim _{N \rightarrow \infty} \frac{1}{N} \sum_{n=0}^{N-1} p\left(T+n / f_{F}\right) .
$$

Here, $T$ refers to the phase-time measured from the starting time of an actuation pulse (see Figure 7). To avoid phase ambiguity, $T$ is not bounded within 0 and $1 / f_{F}$ in the above definition, so that $\tilde{p}$ itself is periodic.

Figure 8 shows the wave component of NFP at $x=2 D$ for forcing at $S t_{D F}=0.02$. Each actuation pulse is seen to generate two well-defined compact waves, both with a positive excursion preceding a negative one. The duration of the compact phase-averaged flow response is almost an

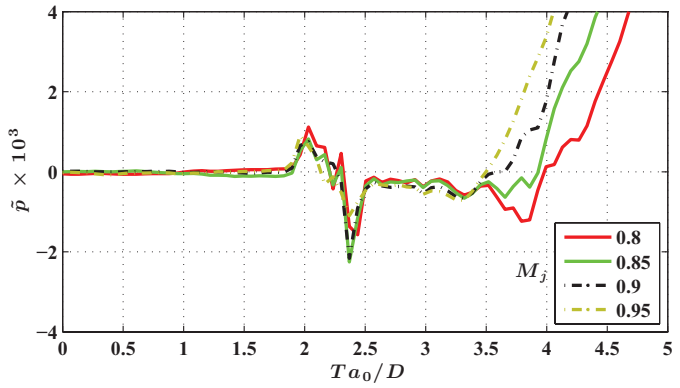

(a)

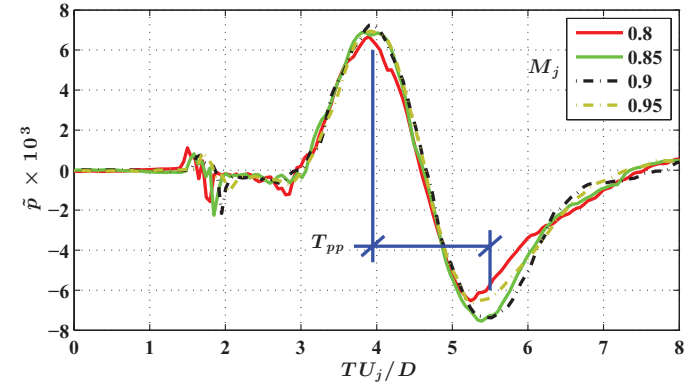

(b)

FIG. 8. Impulse response (with $S t_{D F}=0.02$ ) of NFP at $x=2 D$ with two different phase-time scalings to highlight the (a) acoustic (phase time scaled by ambient speed of sound), and (b) hydrodynamic components (phase time scaled by $U j$ ). 


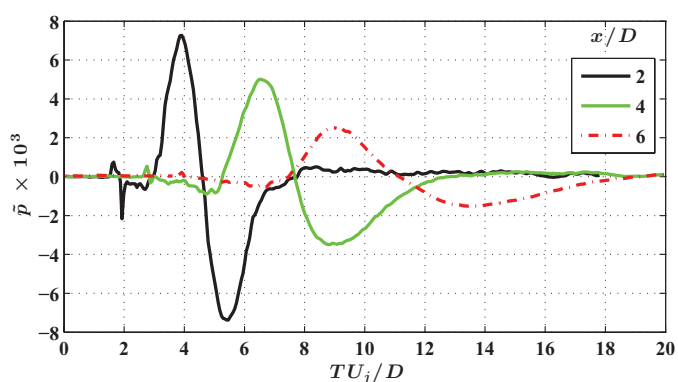

(a)

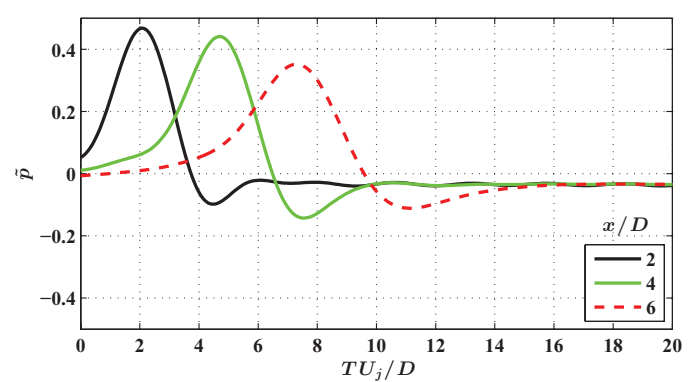

(b)

FIG. 9. Axial evolution of the wave pressure signature of impulsive forcing in the $M_{j}=0.9$ jet. (a) Experimental observation. (b) PSE model prediction.

order-of-magnitude smaller than the forcing period, validating the choice of this periodic forcing for studying the impulse response.

The earlier but smaller wave is the actuator "self-noise" traveling directly to the sensor without being modulated by the flow. This is revealed by the collapse of these signatures irrespective of $M_{j}$ once the phase-time is normalized to $T a_{0} / D$ in Figure 8(a). The distance from the nozzle exit to the sensor is $2.17 D$, which agrees with the observed time of arrival. Phase-locked schlieren imaging has demonstrated that each actuator pulse generates a compression wave ${ }^{9}$ that is discernible near the nozzle, and this is being captured by the sensors in the near-field. The actuator self-noise dissipates quickly, becoming less distinct after the phase-averaging process at the downstream sensors (see Figure 9(a)). The precise shape of the signature cannot be resolved within the bandwidth of the microphones used, but a negative excursion is seen to trail the compressive wave front resulting in zero-net value.

The second compact wave generated by each actuation is much stronger, has very different characteristics compared to the first wave, and is the signature of a vortex-ring-like structure generated by the axisymmetric forcing. This is a hydrodynamic response, as evidenced by the collapse of the curves for different Mach numbers in Figure 8(b) when the phase-time $T$ is normalized to $T U_{j} / D$, and the wave pressure $\tilde{p}$ is normalized by the nozzle exit dynamic head.

The temporal persistence, $T_{p p}$, of the wave response recorded at a sensor is defined as the time from the positive to the negative peak (see Figure 8(b)). This is expected to be proportional to the local size of the structure generated by the plasma actuation. It has been mentioned previously that the convective speed, $U_{c}$, of the hydrodynamic pressure field scales with $U_{j}$. Thus, the inverse scaling of $T_{p p}$ with $U_{j}$ is indicative of the hydrodynamic nature of the wave. The time-of-arrival observed over the range of operating conditions scales inversely with the nozzle exit velocity $U_{j}$, and hence with $U_{c}$. The leading positive excursion is the compressive front associated with the vortex ring (large scale structure) whose core manifests in the trailing negative excursion. The minor amplitude discrepancies across different operating conditions can be expected from the corresponding variations noted for the unforced jets in Figure 4, as well as variations in the forcing conditions.

Figure 9(a) investigates the axial evolution of the impulse response. As expected from the above description of large-scale structures seeded by the impulse, the convective arrival of the impulse response occurs at later times for downstream sensors. The temporal persistence increases with downstream distance; this is analyzed below in detail. The amplitude of the response also decreases with downstream distance in the range depicted. A part of this damping can be explained by the downstream evolution of the linear instability waves (see Figure 6(b)) - this aspect is captured in the linear PSE model predictions of the impulse response juxtaposed in Figure 9(b) and discussed in Sec. V B. Additional contributions to the damping are factors that are outside the scope of the model, viz., nonlinearities and relatively severe non-parallel effects at low frequencies (see Sec. V B).

Figure 10 focuses attention on the axial evolution of the persistence (or compactness) parameter, $T_{p p}$. The width of the shear layer scales almost linearly with $x$ up to the end of the potential core. ${ }^{37}$ 


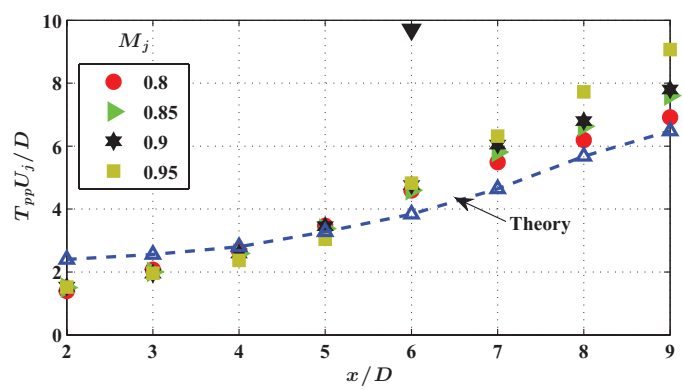

FIG. 10. Axial evolution of the peak-to-peak time in the impulse response of NFP. The inverted triangle marks the approximate axial location of the potential core end.

The corresponding increase in $T_{p p}$ constitutes further evidence that the perturbation seeded by the impulse develops into a large-scale structure that grows with the growing shear layer. The collapse of the normalized $T_{p p}$ values for different $M_{j}$ 's also supports this view. A least-squares line-fit of the $T_{p p}$ values observed over the range $2 \leq x / D \leq 6$ yields

$$
T_{p p} U_{j} / D=0.78 x / D-0.29
$$

with coefficient of determination $R^{2}=0.94$. Beyond $x \approx 6 D$, the $T_{p p}$ values depart from the initial linear behavior. This is linked to the merger of the annular shear layer, in the average, at the end of the potential core which causes distinct changes in LSS dynamics. In addition, the centerline velocity starts to decrease beyond this point, which invalidates the scaling of $T_{p p}$ with the constant $U_{j}$. The theoretical curve in Figure 10 is obtained by PSE in Sec. V B.

The compactness of the wave component of impulse response implies the presence of a broad range of frequencies. Prior to spectral analysis, the infinite-time-horizon impulse response is composed by zero-padding the $S t_{D F}=0.02$ response as

$$
\tilde{p}_{0}(T):= \begin{cases}\tilde{p}\left(T^{\prime} ; f_{F, 0}\right), & \text { if } 0 \leq T^{\prime} \leq 1 / f_{F, 0}, \\ 0, & \text { otherwise. }\end{cases}
$$

Here $f_{F, 0}$ is the forcing frequency corresponding to $S t_{D F}=0.02$. The PSD of $\tilde{p}_{0}$ (arbitrarily scaled to counteract the zero-padding) is presented in Figure 11 for the $M_{j}=0.9$ jet at two representative axial stations. For comparison, the corresponding PSDs of the unforced jet are overlaid. At both stations, the spectrum of the impulse response peaks at lower frequencies compared to the spectrum of the unforced jet.

To quantify this shift in spectral peak, we start by denoting the spectral peak of the phaseaveraged impulse response by $\widetilde{S t} t_{D}^{\max }$. A least-squares linear fit of its reciprocal in the range $2 \leq x / D$

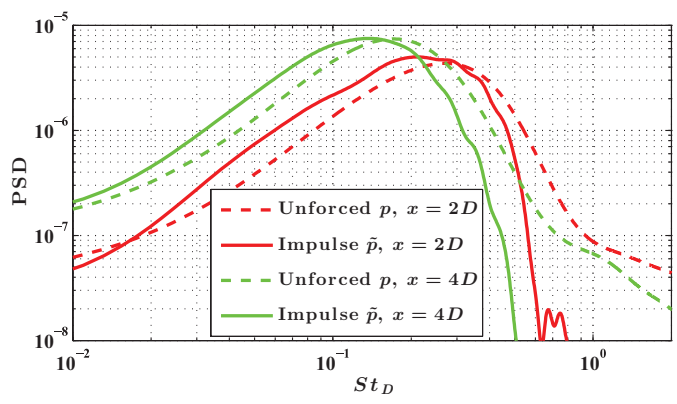

FIG. 11. Arbitrarily scaled spectra of phase-averaged impulse response at two axial stations in the $M_{j}=0.9$ jet compared with corresponding PSDs of the unforced jet. 
$\leq 6$ for all the four $M_{j}$ 's yielded

$$
\frac{1}{S t_{D}^{\max }}=1.90 x / D+0.37
$$

with coefficient of determination $R^{2}=0.91$. This result is to be compared with the corresponding line-fit for the spectral peak of unfiltered pressure in unforced jets in Eq. (3).

Assuming that the plasma breakdown is truly impulsive with equal energy in all frequencies, the spectrum of $\tilde{p}$ may be identified as the Bode diagram of the jet. In this scenario, its comparison with the spectrum of the unforced jet may elucidate the frequency content of the natural disturbances imposed on the jet, presumably at the nozzle lip. The most amplified disturbance in the initial shear layer $^{38}$ is expected to be near $S t_{D} \approx 5$ based on estimates of its momentum thickness for the jets under consideration. ${ }^{7}$ However, this frequency is well beyond the limit imposed on the analysis by the actuator self-noise being of finite temporal duration at the measurement stations. This noise is discernible in the secondary peaks near $S t_{D} \approx 0.7$ in the spectrum of the impulse response at $x=2 D$.

\section{B. Prediction of impulse response from linear PSE analysis}

The linear PSE modes represent the harmonic input/output response of the jet - given the turbulence spectrum at the initial station, one has a theoretical prediction of the amplitude and phase of the fluctuations at all downstream stations. In linear systems theory, the impulse response is retrieved by an inverse Fourier transform of the harmonic response. The forcing delta function is addressed by equalizing the amplitudes and phases of the different frequency modes at the forcing location. It will be recalled that only the Kelvin-Helmholtz mode of instability is used as the initial condition of the PSE. Thus, the additional implicit assumption in the model is that the impulsiveness of the perturbation applies not only to the entire fluctuation but also to the $\mathrm{K}-\mathrm{H}$ mode in isolation. Figure 7 has indicated that the NFP response to actuation is significantly larger than the hydrodynamic fluctuations in the unforced jet. Thus, for the purposes of comparison with the phase-averaged wave signature, the LAFPA forcing is modeled as the impulse response of the mean unforced jet.

The experiments reported here were conducted with the axisymmetric mode of forcing (all LAFPAs firing simultaneously). Large-eddy simulations ${ }^{39}$ indicate that the seeded flow perturbations are azimuthally localized close to the actuators. However, these simulations, as well as phase-locked schlieren experiments, ${ }^{9}$ display azimuthally coherent ring-like structures within a few jet diameters. Prior to this merger, the azimuthal modal content of the fluctuations is $\{0, \pm 8, \pm 16, \ldots\}$ with eight LAFPAs firing. Linear stability theory predicts that all azimuthal modes apart from 0,1 , and 2 are damped in the axial domain of interest (see Figure 6(a)). Thus, the model of the impulse response will include the sole unstable excited PSE mode, viz., $m=0$. PSE models have been proposed for serrated nozzles that linearize about the non-axisymmetric mean flow. ${ }^{40}$ Although this technique may be fruitful for the present application owing to the obvious similarities, it is beyond the scope of this work.

As per the above discussion, the lip-line pressure components of the $m=0$ PSE modes at the most upstream station (hence at $x=0.5 D, r=0.5 D$ ) are normalized to unity. This is done for all PSE modes of Strouhal number up to 0.4 in steps of 0.025 - stable marching of PSE modes with frequencies below 0.025 require axial steps that are longer than the PIV domain, and Figure 6(a) has indicated that frequencies higher than 0.4 are damped at the initial station. Subsequently, the temporal Fourier transform indicated in the PSE ansatz of Eq. (1) is performed. Finally, the fluctuations are re-scaled to simulate a unit pressure impulse at the lip.

The impulse response predicted by the PSE model at the location of the pressure sensors is shown in Figure 9(b). The signals compare favorably with the experimental results in Figure 9(a). The general shapes of the curves, and the trends in downstream evolution of the response are captured quite well. The peak-to-peak times $T_{p p}$ are evaluated from the modeled wave shapes and are presented as the theoretical curve in Figure 10. The match with experimental observations is quite close in this regard. 

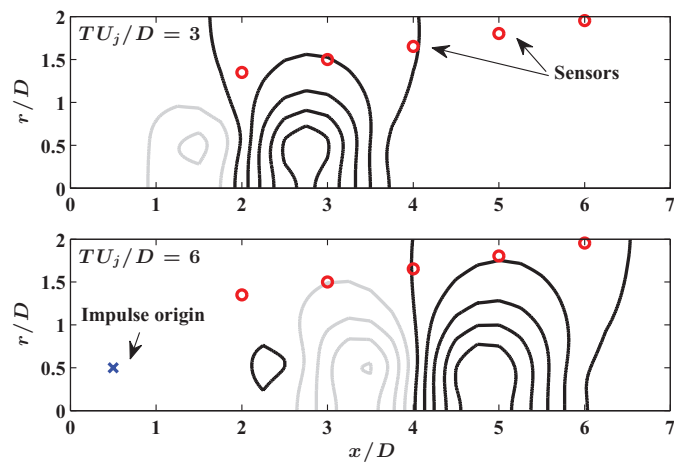

FIG. 12. Pressure fluctuations at two instants from PSE model of impulsive forcing. Equi-spaced positive and negative contours are in black and grey, respectively.

The linear PSE model appears to predict the essential aspects of the impulse response of the jet. The points of disagreement in Figures 9 and 10 illustrate the properties of the PSE approach. The pulses appear to arrive at the virtual NFP sensors in Figure 9(b) about $1.8 \mathrm{D} / U_{j}$ earlier compared to the experimental observation in Figure 9(a). This is explained by recalling that the initial condition of the model is a pressure peak at $x=0.5 D$. This ignores the time of convection $\left(\approx 0.7 D / U_{j}\right)$ from the actuators just upstream of the nozzle exit, as well the rise time of the waveform $\left(\approx 1.1 D / U_{j}\right.$ near the nozzle) observed in Figure 9(a).

The temporal scale of the impulse response experimentally observed at the microphone at $x$ $=2 D$ is shorter by about $60 \%$ than the PSE prediction (see Figure 10). This discrepancy is much less pronounced at downstream stations. As discussed above, the PSE model cannot simulate the initial azimuthal localization of the forcing effect. Moreover, vorticity and entropy modes that are not included in the model, may be significantly energetic near the plasma actuator but are damped further downstream. The slowly-varying base flow assumption in PSE is tenuous at low frequencies that are dominant further downstream, ${ }^{22}$ and this causes the discrepancy in $T_{p p}$ at the downstream microphone stations (see Figure 10).

Figure 12 shows the pressure fluctuation fields from PSE at two representative instants after an impulsive event. The leading positive pressure pulse can be traced back to the impulsive forcing at zero phase-time (not shown). The initial strength of the trailing negative pressure excursion is much lower compared to the leading one, but Figure 9(a) indicates that this is an erroneous prediction. The cause of the mismatch is the neglect of the modulation of the impulsive perturbation by the jet between its actual initiation just upstream of the nozzle exit and its modeled location at $x=0.5 D$.

The PSE serves as a useful tool for efficient predictions, so that a certain approximation is allowable. Keeping this in mind, the correspondence between theory and observation is reasonably close to feel confident that the linear PSE model is capturing the correct mechanism.

\section{THE JET RESPONSE TO PERIODIC IMPULSES}

Section V has described the response of the turbulent jet to a single impulse. It was asserted that, although the actual forcing was periodic, the $S t_{D F}$ of 0.02 was low enough to be immaterial to the result. In this section, we elaborate on this premise by investigating the jet response to periodic impulsive forcing at higher frequencies. Section VI A begins the study with a discussion of the effect of increasing $S t_{D F}$ on the wave component of pressure, $\tilde{p}$, in the phase-time domain. Section VI B follows this up with a description of the changes in the spectral domain, both for $\tilde{p}$ and the unfiltered pressure. Section VI C concludes with an analysis of the effect of forcing periodicity on the mean-squared near-field pressure. The common theme in these three approaches is the establishment of a quasi-linear property of the jet response to periodic impulses. This follows the success of linear PSE in predicting the characteristics of the unforced jet as well as the impulse response of the jet in Secs. IV B and V B. 


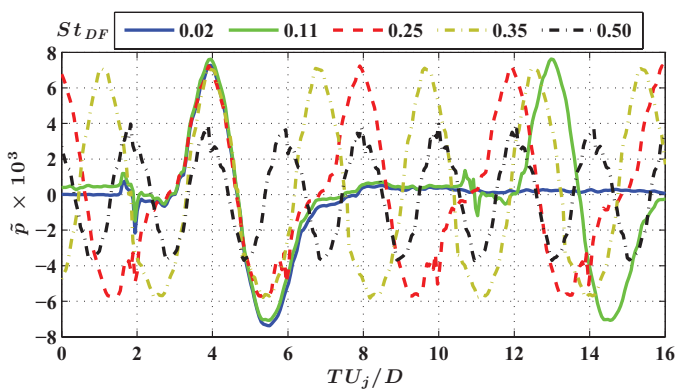

(a)

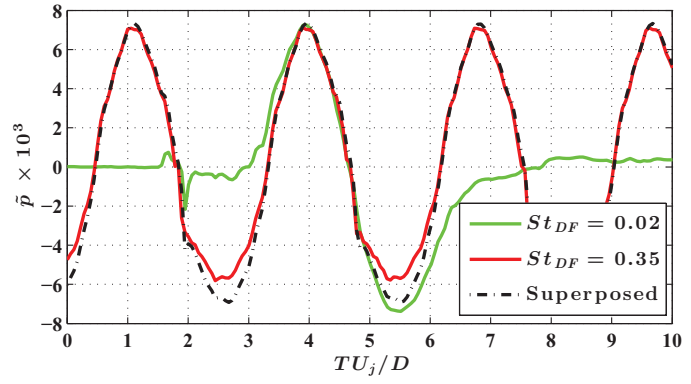

(b)

FIG. 13. (a) Effect of periodicity of impulsive forcing on wave component of NFP in $M_{j}=0.9$ at $x=2 D$. (b) Modeling periodic forcing response by superposition of impulse response under the same conditions.

\section{A. Wave component of pressure in phase-time domain}

Phase-averaging analysis has elucidated the impulse response of the jet in Sec. V A. The impact of periodicity of the impulsive actuation is now studied by employing a range of forcing frequencies covering the characteristic frequencies of the jet. Figure 13(a) depicts the wave response of the NFP in the Mach 0.9 jet at $x=2 D$ for some salient $S t_{D F}$ 's. The case of $S t_{D F}=0.02$ was introduced in Figure $8(\mathrm{~b})$, and it has been extensively discussed in Sec. V. The fundamental response is seen to remain unchanged up to $S t_{D F}=0.11$. The periodicity only results in more pulses being captured within the averaging window. This is the justification for identifying the jet response with $S t_{D F}$ $\leq 0.1$ as the impulse response. Increasing $S t_{D F}$ to 0.25 results in an almost sinusoidal response, but the fundamental shape of the positive peak still remains unchanged. However, the amplitude of the negative excursion is reduced, and the actuator self-noise of the succeeding pulse is also detectable in this region of the hydrodynamic fluctuation. The pulse build-up has sharpened at the higher $S t_{D F}$ of 0.35 , although the peak-to-peak time, $T_{p p}$, as well as the amplitude, have not been significantly affected. By $S t_{D F}=0.50$, distinct reductions in both $T_{p p}$ and the amplitude are observed.

The impulse response has been modeled with relative fidelity with linear PSE. To assess the linearity of the jet response to periodic impulses, the wave component of response at a given forcing frequency, $f_{F}$, is approximated by linear superposition of the infinite-time-horizon impulse response defined in Eq. (7),

$$
\tilde{p}\left(T ; f_{F}\right) \approx \sum_{n=-\infty}^{\infty} \tilde{p}_{0}\left(T+n / f_{F}\right) .
$$

Figure 13(b) demonstrates that the result of the above superposition closely resembles the actual wave component of response at $S t_{D F}=0.35$. This indicates that the seeded LSS in the flow are interacting quasi-linearly with the succeeding LSS in the wave train. The main discrepancy is the slight over-prediction of the amplitude at the negative peak. The validity of this quasi-linearity argument will be demonstrated over a wide range of $S t_{D F}$ 's in Secs. VI B and VI C.

The next step is the quantification of the variation of the temporal persistence parameter, $T_{p p}$, of the wave response with $S t_{D F}$. The $\tilde{p}$ response curves are not very smooth in some cases owing to the superposition of the actuator self-noise. Wavelet filtering is performed to smooth the curves while maintaining its compact shape. ${ }^{41}$ The fourth-order Paul wavelet was chosen as the mother, since its imaginary part resembles the impulse response, and wavelets with amplitude less than $25 \%$ of the maximum amplitude were rejected prior to the reconstruction. The results presented here are quite insensitive to the precise threshold, since a first-order parameter is being extracted.

Values of $T_{p p}$ determined from the wavelet-filtered responses are plotted in Figure 14(a) for the sensor at $x=2 D$. The initial flatness of the curves reflects the invariant nature of the fundamental response at these low $S t_{D F}$ 's. Moreover, as expected from Figure $8(\mathrm{~b})$, the values of $T_{p p} U_{j} / D$ are the same, within the measurements accuracies, over the tested range of nozzle exit velocities. Be- 


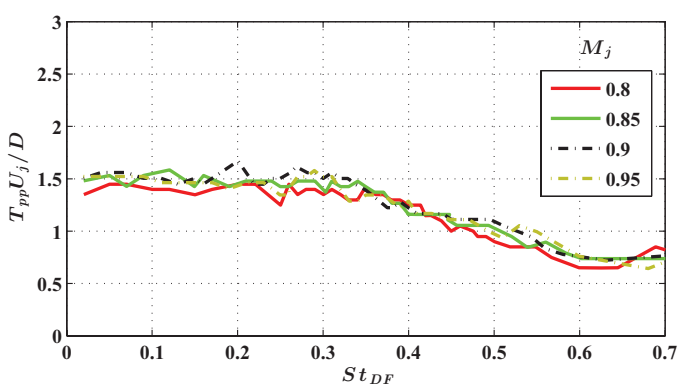

(a)

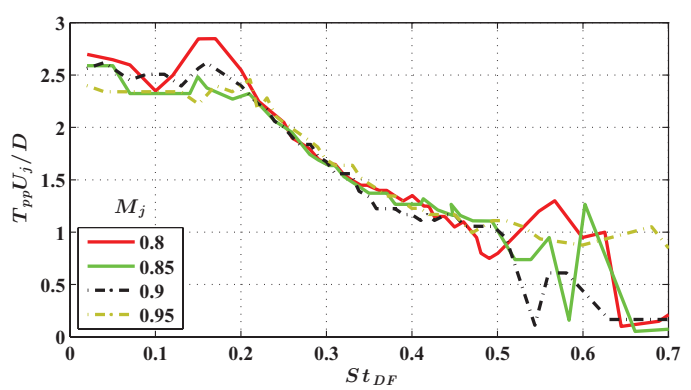

(b)

FIG. 14. Time between the positive and negative peaks of the wave component of NFP response. (a) $x=2 D$. (b) $x=4 D$.

yond a certain shoulder in the curves near $S t_{D F} \approx 0.33$, there is a gradual decrease of $T_{p p}$. This is also discernible in Figure 13(a) and is interpreted as the onset of interaction between neighboring seeded structures as the time period of forcing reduces below the temporal extent of the impulse response. In a re-plotting of these curves with $2\left(T_{p p} U_{j} / D\right) S t_{D F}$ vs. $S t_{D F}$ (not shown here), these regions of the curves were found to be quite flat at unity. Thus, the response over this range of $S t_{D F}$ 's is such that $T_{p p} \approx 0.5 / f_{F}$. Beyond $S t_{D F} \gtrsim 0.6$, the hydrodynamic response cannot be reliably separated from the actuator self-noise (see Sec. VI B), so that the analysis of $T_{p p}$ cannot be continued.

Figure 14(b) demonstrates that the $T_{p p}$ curves for the $x=4 D$ station have a similar behavior. The constant $T_{p p}$ value at the lowest $S t_{D F}$ 's is increased, as can be expected from Figure 10. The decay portions of the curves are universal as explained for Figure 14(a) above. Thus, the increase in the initial $T_{p p}$ brings the shoulders of the curves to a lower $S t_{D F}$ of around 0.2. The analysis of $T_{p p}$ has to be discontinued for $S t_{D F} \gtrsim 0.5$ at this observation station.

\section{B. Response in the spectral domain}

The quasi-linearity of the jet response to periodic impulsive forcing has been demonstrated above in the phase-time domain for $S t_{D F}=0.35$. The same argument is made from a spectral perspective for a range of $S t_{D F}$ 's here. For the periodic impulsive forcing considered here, the energy spectral density (ESD) per pulse is more suitable than the PSD. For the infinite-time-horizon impulse response $\tilde{p}_{0}(T)$ defined in Eq. (7), one has

$$
\operatorname{ESD}\left[\tilde{p}_{0}\right](f):=\left|\int_{0}^{\infty} \tilde{p}_{0}(T) \mathrm{e}^{-2 \pi \mathrm{i} f T} \mathrm{~d} T\right|^{2} .
$$

The ESD is presented in Figure 15 for the $M_{j}=0.9$ jet at $x / D=2$ and 4 . These curves differ from the PSD of Figure 11 in that they are not scaled arbitrarily any more.

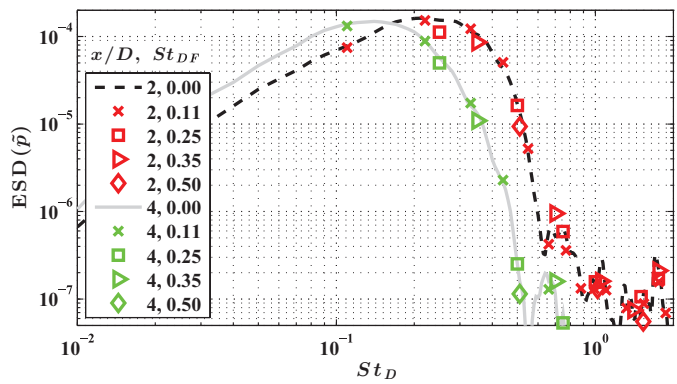

FIG. 15. Energy spectral density of phase-averaged NFP at two axial stations in the $M_{j}=0.9$ jet with changing periodicity of impulsive forcing. $S t_{D F}=0$ refers to the infinite-time-horizon impulse response. Markers indicate only the harmonic peaks in the discrete energy spectra for periodic impulses, the amplitude at intervening frequencies being below the noise floor. 


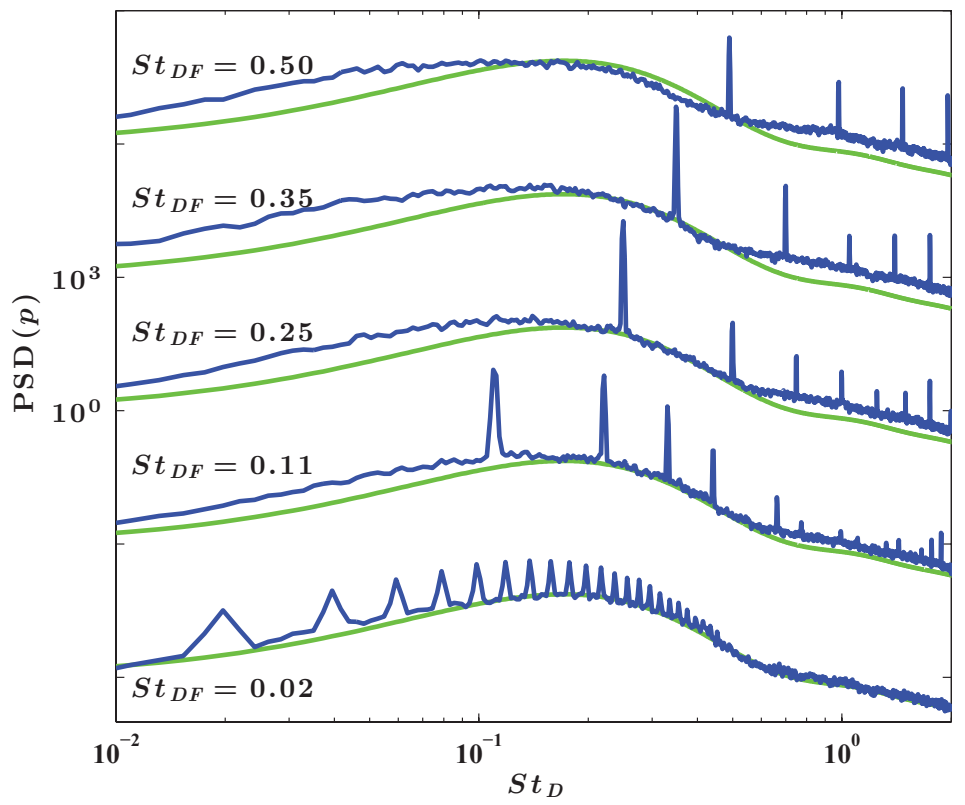

FIG. 16. Spectra of unfiltered pressure measured at $x=4 D$ in the $M_{j}=0.9$ jet forced at several frequencies compared with the unforced case.

For the phase-averaged periodic impulsive forcing with frequency $f_{F}$ defined in Eq. (5), the ESD per pulse is

$$
\operatorname{ESD}\left[\tilde{p}\left(\cdot ; f_{F}\right)\right](f)=\lim _{K \rightarrow \infty}\left|\frac{1}{K} \int_{0}^{K / f_{F}} \tilde{p}\left(T ; f_{F}\right) \mathrm{e}^{-2 \pi \mathrm{i} f T} \mathrm{~d} T\right|^{2} .
$$

As the number of periods $K$ increases, the spectral tones become sharper. Figure 15 displays the tonal levels obtained with $K=100$ for four of the $S t_{D F}$ cases depicted in Figure 13(a). At frequencies in between the tones, the ESD falls below the noise floor dictated by the numerics. The tonal levels are seen to closely follow the curves for $\operatorname{ESD}\left[\tilde{p}_{0}\right]$ at both measurement locations. For a linear system, the tonal levels should exactly follow the curves ${ }^{42}$ since the superposition in Eq. (9) is an exact model in that case. Thus the minor disagreements observed here implicate nonlinearities. The match between the tonal levels and the impulse response's spectrum is better at $x=4 D$. This mirrors the corresponding improvement of linear PSE predictions at downstream locations (see Figures 9 and 10). The tones for the $S t_{D F}=0.02$ case are not shown since they necessarily fall exactly on the $\operatorname{ESD}\left[\tilde{p}_{0}\right]$ curves. The overall agreement of the results with expectations for linear systems further validate the quasi-linearity hypothesis.

The actuator self-noise appears in the ESD around 0.7 and 0.6 at $x / D=2$ and 4 , respectively. This explains why the peak-to-peak time, $T_{p p}$, of the phase-averaged signals in Figure 14 becomes uncertain for $S t_{D F}$ 's approaching these values.

The preceding spectral analysis of the phase-averaged pressure masks the broadband modifications generated by forcing. Hence, the PSD of the unfiltered NFP is explored for some representative forcing cases in Figure 16. The forcing tones and their harmonics, that were studied in isolation above, are now seen to rise several decades above the broadband level. The fundamental tone is strongest at $S t_{D F}=0.25$ and 0.35 , which are close to the jet column mode instability, with a decrease in tonal levels at both lower and higher $S t_{D F}$ 's.

Although not shown here, the PSD was also computed from the phase-averaged response. This is akin to the ESD of these signals discussed above and differs only in the normalization. The resulting discrete spectra have tonal levels that closely match those observed for the PSD of the unfiltered pressure in Figure 16. This indicates that the statistics are sufficiently converged so that the two operations - averaging and spectrum computation - are effectively commutable. 


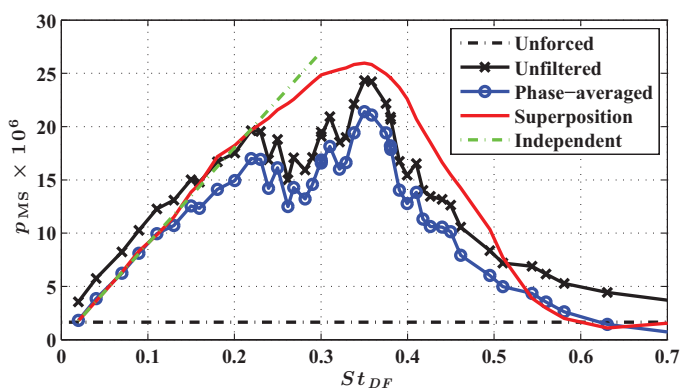

(a)

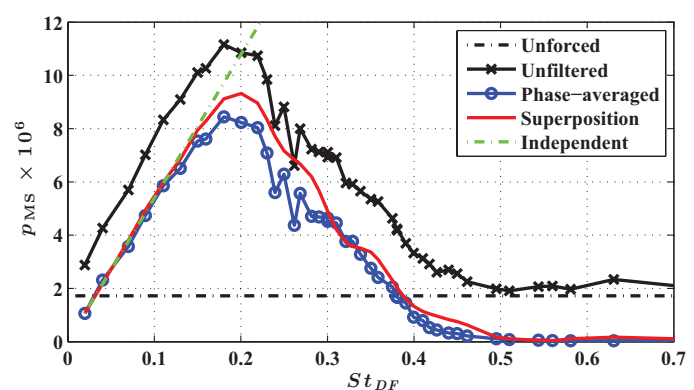

(b)

FIG. 17. Comparison of mean-square NFP analyzed with different methods for the $M_{j}=0.9$ jet at two observation stations. Note the difference in ordinate scales. (a) $x=2 D$. (b) $x=4 D$.

The broadband spectral level does not depart appreciably from the unforced spectrum at the lowest $S t_{D F}=0.02$, but significant amplification is observed at the three intermediate forcing frequencies presented. There is broadband amplification as well as attenuation at different spectral regions with the highest $S t_{D F}$ of 0.50 . These broadband changes cannot be explained by the aforementioned phase-averaging and superposition techniques. The implication is that nonlinearities serve to excite the broad range of frequencies for forcing near the jet column mode. An extensive earlier study has reported the spectral properties of the unfiltered near-field pressure, as well as its azimuthal Fourier decomposition, for the forced $M_{j}=0.9$ jet. $^{11}$

\section{Energy of pressure response in forced jet}

The trends in the response of the jet near-field pressure to periodic impulsive forcing have been discussed in Secs. VI A and VI B using some salient forcing Strouhal numbers. Here, these characteristics are investigated for a larger set of $S t_{D F}$ 's with the mean-square of the pressure fluctuations, $p_{\mathrm{MS}}$ (alternatively termed "energy"), to compress the information. Both the unfiltered pressure and its wave component are analyzed.

Figure 17 shows the mean-square of the unfiltered pressure in the $M_{j}=0.9$ jet at $x / D=2$ and 4. The energy of the jet response resulting from plasma actuation is frequency-dependent, which is commonly attributed to the jet column mode instability (this point is elaborated below). Increasing the $S t_{D F}$ causes a rapid increase in $p_{\mathrm{MS}}$ followed by a gradual decrease. The $S t_{D F}$ corresponding to the peak response decreases with downstream distance of the measurement location, mirroring the decrease in $S t_{D}^{\max }$ in the unforced jet (see Figure 5(b)). The mean-square NFP in the unforced jet is shown for reference.

The energy is also calculated for the wave component of pressure signals, taking care to truncate the calculation to exactly one forcing period - this is denoted $\tilde{p}_{\mathrm{MS}}$. The curve for $\tilde{p}_{\mathrm{MS}}$ demonstrates almost a fixed decrement from $p_{\mathrm{MS}}$ over most of the $S t_{D F}$ range. If there were no broadband modification with forcing, the difference between the two curves would equal the $p_{\mathrm{MS}}$ of the unforced jet (see Figure 16).

The quasi-linearity of the phase-averaged pressure response to periodic impulsive forcing has been demonstrated in Secs. VI A and VI B; this is reassessed with the energy metric here. The superposition in Eq. (9) is performed over the range of $S t_{D F}$ 's, and the mean-squares computed from these are overlaid in Figure 17. The comparison with $\tilde{p}_{\mathrm{MS}}$ confirms the observations in Figure 15, while quantifying the discrepancies that were difficult to discern in the log-scale plot. As expected, the match at $x=2 D$ is very good at low-frequencies. At frequencies near the peak response and beyond, the discrepancy is more pronounced. At $x=4 D$ though, the superposition provides a very good match with $\tilde{p}_{\text {MS }}$ over most of the forcing frequency range shown.

The constancy of the peak-to-peak time, $T_{p p}$, at the lower frequencies in Figure 14 was understood to signify the lack of interaction between the periodically seeded structures. This conjecture can be further evaluated with the energy metric in the following manner. The $\tilde{p}$ for the lowest 
frequency has compact support within one forcing period. Then, assuming independent addition of successive structures all with the same compact signature, the mean-square pressure should be directly proportional to the forcing frequency. This trend is indicated in Figure 17, and it is indeed seen to model the $p_{\mathrm{MS}}$ from superposition up to $S t_{D F} \approx 0.25$ and 0.15 at $x / D=2$ and 4 , respectively.

Structure interaction begins beyond these threshold forcing frequencies at the corresponding axial stations, so that independent addition is an incorrect model. Figures 13(a) and 14 reveal that the interaction does not modify $T_{p p}$ or the peak amplitudes initially. Instead, the relaxation of the pressure field is hastened primarily. Consequently, the mean-square pressure keeps on increasing with increasing $S t_{D F}$. However, this monotonic trend is arrested at $S t_{D F}$ 's that approximately correspond to the shoulders where $T_{p p}$ starts to decrease in Figure $14-S t_{D F}$ 's of 0.33 and 0.2 at $x / D=2$ and 4 , respectively. Figure 13(a) shows that the slope of the fall from the positive peak to the negative one remains unchanged to much higher frequencies. Thus, the decrease in $T_{p p}$ is indicative of a reduction in the amplitude of the peaks of the wave component of signature. An intuitive understanding is that the neighboring structures inhibit each other's growth.

Many earlier experiments have observed the existence of an optimum frequency for mixing enhancement in jets forced with LAFPAs. ${ }^{3,6,8,10}$ This has been explained as a resonance with the jet column mode, which, in turn, is taken to imply the natural frequency of the undulation of the jet column or potential core. In this research, the observations in the phase-averaged near-field pressure response afford another perspective on this behavior based on interactions of the periodically seeded structures. The impulse response has been modeled relatively well with linear stability theory that addresses the K-H instability, and the response to periodic impulses has been quasi-linearly related to the impulse response. The jet column mode has previously been explained in terms of the inflectional instability of the shear layer ${ }^{43}$ and the present findings support this view.

\section{DISCUSSION AND CONCLUSION}

The near-field pressure has been probed to investigate the response of subsonic jets to forcing with LAFPAs. These actuators have demonstrated superior control authority on high-speed turbulent jets over a wide range of practical operating conditions. Four subsonic Mach numbers $\left(M_{j}=0.80\right.$ $0.95)$ are investigated here to discern scaling laws for the response of the jet. The arc breakdown imparts an impulsive perturbation to the flow each time an actuator is switched on. Eight LAFPAs uniformly arrayed around the periphery of the nozzle exit are operated in phase to simulate axisymmetric forcing. Phase-averaging of the pressure signal with respect to the actuator signal has been leveraged to separate the relevant features of the response from the background turbulence.

At forcing Strouhal numbers less than 0.1, the phase-averaged pressure signature displays a compact sinusoidal wave with one positive excursion preceding one negative excursion. Scaling of the parameters of this wave with the nozzle exit velocity indicates that this response is hydrodynamic. There is a second compact wave in the phase-averaged pressure signature - but this is much smaller in amplitude, decreases rapidly with downstream distance from the nozzle, and is determined to be the actuator self-noise traveling with the ambient speed of sound. Since the time scale of the hydrodynamic response is much shorter than the forcing period employed to generate it, this is deemed the impulse response. The conclusion is that each actuator impulse creates a perturbation which is amplified by the flow and develops into a large scale vortex-ring-like structure. The negative peak in the phase-averaged near-field pressure is associated with the vortex core, whereas the leading compressive front appears as the preceding positive peak in the pressure signature. Owing to the impulsive nature of the actuator, this appears to be a very robust and reliable method of generating a single structure with precise timing in the highly turbulent flow.

Linear parabolized stability theory is applied in a novel manner to model the impulse response using merely the mean axial velocity data of the unforced jet from a PIV assay. The predictions approach reasonably close to the experimentally determined phase-averaged signature, pointing to a quasi-linear mechanism of plasma actuation.

Beyond a threshold forcing frequency that is inversely proportional to the downstream distance of the measurement station, the periodically seeded structures begin interacting with each other. However, the structure interaction appears to be quasi-linear - the phase-averaged wave shape can 
be reconstructed reasonably accurately by linear superposition of the impulse response repeatedly shifted by the forcing periodicity. This mirrors the agreement of linear PSE predictions of the impulse response with experimental observations. In the spectral domain, the quasi-linearity is manifested in relatively accurate prediction of the fundamental and harmonic tonal levels from knowledge of the impulse response. However, there are broadband changes in the pressure spectrum at intermediate frequencies which implicate additional nonlinear mechanisms.

Our previous investigations have established that at high enough forcing Strouhal numbers, the mean-square near-field pressure decreases below that observed in the unforced jet, paralleling the significant mitigation of the far-field noise. This benefit of LAFPA application is of urgent practical interest to the aviation community. However, the phase-averaging technique pursued here is inappropriate for studying this important forcing regime since the hydrodynamic pressure fluctuations cannot be reliably distinguished from the actuator self-noise at these higher Strouhal numbers.

In summary, the impulse response of turbulent jets has been studied using LAFPAs. Important insight is gleaned regarding the quasi-linearity of the near-field pressure response with periodic impulsive forcing. In particular, the peak response characterizing the jet column mode is explained using structure interaction. The agreement, albeit qualified, of linear parabolized stability theory with forced jet experiments is also important for physical understanding of the mechanism of plasma actuation.

\section{ACKNOWLEDGMENTS}

The support of this research by the Air Force Office of Scientific Research with Dr. John Schmisseur is greatly appreciated. The PSE code has been developed over the years by Dr. Kristjan Gudmundsson, Dr. Arnab Samanta, Dr. Daniel Rodriguez, and the first author (Aniruddha Sinha), under the guidance of the last author (Tim Colonius) - their contributions are gratefully acknowledged. The article was improved thanks to comments and suggestions from referees.

${ }^{1}$ M. Samimy, I. Adamovich, B. Webb, J. Kastner, J. Hileman, S. Keshav, and P. Palm, "Development and characterization of plasma actuators for high-speed jet control," Exp. Fluids 37, 577-588 (2004).

${ }^{2}$ Y. G. Utkin, S. Keshav, J.-H. Kim, J. Kastner, I. V. Adamovich, and M. Samimy, "Development and use of localized arc filament plasma actuators for high-speed flow control,” J. Phys. D 40, 685-694 (2007).

${ }^{3}$ M. Samimy, J.-H. Kim, J. Kastner, I. Adamovich, and Y. Utkin, "Active control of high-speed and high-Reynolds-number jets using plasma actuators," J. Fluid Mech. 578, 305-330 (2007).

${ }^{4}$ S. Crow and F. Champagne, "Orderly structure in jet turbulence,” J. Fluid Mech. 48, 547-591 (1971).

${ }^{5}$ A. Michalke, "Survey on jet instability theory," Prog. Aerosp. Sci. 21, 159-199 (1984).

${ }^{6}$ M. Samimy, J.-H. Kim, M. Kearney-Fischer, and A. Sinha, "Acoustic and flow fields of an excited high Reynolds number axisymmetric supersonic jet," J. Fluid Mech. 656, 507-529 (2010).

${ }^{7}$ M. Kearney-Fischer, J.-H. Kim, and M. Samimy, "Control of a high Reynolds number Mach 0.9 heated jet using plasma actuators," Phys. Fluids 21, 095101 (2009).

${ }^{8}$ J.-H. Kim, J. Kastner, and M. Samimy, "Active control of a high Reynolds number Mach 0.9 axisymmetric jet," AIAA J. 47, 116-128 (2009).

${ }^{9}$ C. Hahn, M. Kearney-Fischer, and M. Samimy, "On factors influencing arc filament plasma actuator performance in control of high speed jets," Exp. Fluids 51, 1591-1603 (2011).

${ }^{10}$ M. Samimy, J.-H. Kim, M. Kearney-Fischer, and A. Sinha, "High-speed and high Reynolds number jet control using localized arc filament plasma actuators," J. Propul. Power 28, 269-280 (2012).

${ }^{11}$ A. Sinha, K. Kim, J.-H. Kim, A. Serrani, and M. Samimy, "Extremizing feedback control of a high-speed and high Reynolds number jet," AIAA J. 48, 387-399 (2010).

${ }^{12}$ R. E. A. Arndt, D. F. Long, and M. N. Glauser, "The proper orthogonal decomposition of pressure fluctuations surrounding a turbulent jet," J. Fluid Mech. 340, 1-33 (1997).

${ }^{13}$ C. Picard and J. Delville, "Pressure velocity coupling in a subsonic round jet," Int. J. Heat Fluid Flow 21, 359-364 (2000).

${ }^{14}$ L. S. Ukeiley and M. K. Ponton, "On the near field pressure of a transonic axisymmetric jet," Int. J. Aeroacoust. 3, 43-65 (2004).

${ }^{15}$ T. Suzuki and T. Colonius, "Instability waves in a subsonic round jet detected using a near-field phased microphone array," J. Fluid Mech. 565, 197-226 (2006).

${ }^{16}$ C. E. Tinney and P. Jordan, “The near pressure field of co-axial subsonic jets," J. Fluid Mech. 611, 175-204 (2008).

${ }^{17}$ R. Reba, S. Narayanan, and T. Colonius, "Wave-packet models for large-scale mixing noise," Int. J. Aeroacoust. 9, 533-558 (2010).

${ }^{18}$ K. Low, B. El Hadidi, M. Y. Andino, R. Berdanier, and M. N. Glauser, "Investigation of different active flow control strategies for high speed jets using synthetic jet actuators," AIAA Paper No. 2010-4267, 2010.

${ }^{19}$ R. Maury, A. V. G. Cavalieri, P. Jordan, J. Delville, and J.-P. Bonnet, "A study of the response of a round jet to pulsed 
fluidic actuation,” AIAA Paper No. 2011-2750, 2011.

${ }^{20}$ M. Koenig, C. Fourment-Cazenave, P. Jordan, and Y. Gervais, "Jet noise reduction by fluidic injection from a rotating plug," AIAA Paper No. 2011-2899, 2011.

${ }^{21}$ F. P. Bertolotti and T. Herbert, "Analysis of the linear stability of compressible boundary layers using the PSE," Theor. Comput. Fluid Dyn. 3, 117-124 (1991).

${ }^{22} \mathrm{~K}$. Gudmundsson and T. Colonius, "Instability wave models for the near-field fluctuations of turbulent jets," J. Fluid Mech. 689, 97-128 (2011).

${ }^{23}$ D. Rodriguez, A. Sinha, G. Bres, and T. Colonius, "Parabolized stability equation models in turbulent supersonic jets," AIAA Paper No. 2012-2117, 2012.

${ }^{24}$ A. V. G. Cavalieri, D. Rodriguez, P. Jordan, T. Colonius, and Y. Gervais, "Wavepackets in the velocity field of turbulent jets," AIAA Paper No. 2012-2115, 2012.

${ }^{25}$ J. W. Nichols and S. K. Lele, "Global modes and transient response of a cold supersonic jet," J. Fluid Mech. 669, 225-241 (2011).

${ }^{26}$ C. Hahn, "Design and validation of the new jet facility and anechoic chamber," M.S. thesis, The Ohio State University, 2011.

${ }^{27}$ L. C. Cheung and S. K. Lele, "Linear and nonlinear processes in two-dimensional mixing layer dynamics and sound radiation," J. Fluid Mech. 625, 321-351 (2009).

${ }^{28}$ F. Li and M. R. Malik, "On the nature of PSE approximation," Theor. Comput. Fluid Dyn. 8, 253-273 (1996).

${ }^{29}$ F. Coiffet, P. Jordan, J. Delville, Y. Gervais, and F. Ricaud, "Coherent structures in subsonic jets: A quasi-irrotational source mechanism?" Int. J. Aeroacoust. 5, 67-89 (2006).

${ }^{30}$ M. Harper-Bourne, "On modelling the hydrodynamic field of high-speed jets," AIAA Paper No. 2004-2830, 2004.

${ }^{31}$ C. E. Tinney, L. S. Ukeiley, and M. N. Glauser, "Low-dimensional characteristics of a transonic jet. Part 2. Estimate and far-field prediction,” J. Fluid Mech. 615, 53-92 (2008).

32 J. W. Hall, A. M. Hall, J. T. Pinier, and M. N. Glauser, "Cross-spectral analysis of the pressure in a Mach 0.85 turbulent jet," AIAA J. 47, 54-59 (2009).

${ }^{33}$ D. H. Jung, S. Gamard, and W. K. George, "Downstream evolution of the most energetic modes in a turbulent axisymmetric jet at high Reynolds number. Part 1. The near-field region," J. Fluid Mech. 514, 173-204 (2004).

${ }^{34}$ C. E. Tinney, F. Coiffet, J. Delville, A. M. Hall, P. Jordan, and M. N. Glauser, "On spectral linear stochastic estimation," Exp. Fluids 41, 763-775 (2006).

${ }^{35}$ M. Kearney-Fischer, J.-H. Kim, and M. Samimy, “A study of Mach wave radiation using active control,” J. Fluid Mech. 681, 261-292 (2011).

${ }^{36}$ A. K. M. F. Hussain and W. C. Reynolds, "The mechanics of an organized wave in turbulent shear flow," J. Fluid Mech. 41, 241-258 (1970).

${ }^{37}$ V. Fleury, C. Bailly, E. Jondeau, M. Michard, and D. Juve, "Space-time correlations in two subsonic jets using dual particle image velocimetry measurements," AIAA J. 46, 2498-2509 (2008).

${ }^{38}$ K. B. M. Q. Zaman and A. K. M. F. Hussain, “Turbulence suppression in free shear flows by controlled excitation,” J. Fluid Mech. 103, 133-159 (1981).

${ }^{39}$ D. V. Gaitonde and M. Samimy, "Coherent structures in plasma-actuator controlled supersonic jets: Axisymmetric and mixed azimuthal modes," Phys. Fluids 23, 095104 (2011).

${ }^{40} \mathrm{~K}$. Gudmundsson, "Instability wave models of turbulent jets from round and serrated nozzles," Ph.D. dissertation (California Institute of Technology, 2010).

${ }^{41}$ C. Torrence and G. P. Compo, "A practical guide to wavelet analysis," Bull. Am. Meteorol. Soc. 79, 61-78 (1998)

${ }^{42}$ R. Bracewell, The Fourier Transform and its Applications, 3rd ed. (McGraw-Hill, 2000).

${ }^{43}$ R. A. Petersen and M. M. Samet, “On the preferred mode of jet instability," J. Fluid Mech. 194, 153-173 (1988). 